
\title{
3 Research Square \\ Trends in Shaken Baby Syndrome Diagnoses Among Young Children Hospitalized for Abuse
}

Aislinn Conrad ( $\square$ aislinn-conrad@uiowa.edu )

University of lowa https://orcid.org/0000-0002-9446-9410

Brandon Butcher

University of lowa

Resmiye Oral

Dartmouth College Geisel School of Medicine

Megan Ronnenberg

University of lowa

Corinne Peek-Asa

University of lowa

\section{Original Contribution}

Keywords: shaken baby syndrome, abusive head trauma, child abuse, trends, secondary data

Posted Date: April 17th, 2020

DOI: https://doi.org/10.21203/rs.3.rs-22246/v1

License: (c) (i) This work is licensed under a Creative Commons Attribution 4.0 International License.

Read Full License

Version of Record: A version of this preprint was published at Injury Epidemiology on July 19th, 2021. See the published version at https://doi.org/10.1186/s40621-021-00334-w. 


\section{Abstract}

Objective To investigate national trends of SBS diagnosis codes and how trends varied among patient and hospital characteristics. Methods We examined possible and confirmed SBS diagnoses among children age three and younger who were hospitalized for abuse between 1998 and 2014 using a secondary analysis of the National Inpatient Sample, the largest US all-payer inpatient care database ( $\mathrm{N}$ $=52,562)$. A baseline category logit model was used based on a quasi-likelihood approach (QIC) with an independent working correlation structure. Results The rate of confirmed SBS diagnoses increased from 3.8 ( \pm 0.3$)$ in 1998 to $5.1( \pm 0.9)$ in 2005 , and decreased to $1.3( \pm 0.2)$ in 2014. Possible SBS diagnoses were $0.6( \pm 0.2)$ in 1998, and increased to $2.4( \pm 0.4)$ in 2014. Confirmed SBS diagnoses have declined since 2002, while possible SBS diagnoses have increased. Possible SBS diagnoses were more common among urban teaching hospitals and small to medium hospitals than for other hospital types. Conclusions We investigated seventeen-year trends of SBS diagnoses among young children hospitalized for abuse. The discrepancy between trends in possible and confirmed SBS suggests differences in diagnostic norms for SBS and related conditions. Researchers should examine diagnostic processes for SBS and investigate why cases are diagnosed as SBS or a related diagnosis. We propose that researchers and pediatric medical providers agree to a standardized definition and diagnostic guidelines for SBS, much like the AHT guidelines proposed by CDC, which may help reduce discrepancies in diagnosis and improve options for surveillance.

\section{Introduction}

An estimated 1,300 young U.S. children are violently shaken every year, resulting in a unique pattern of neurological injuries referred to as Shaken Baby Syndrome (SBS). ${ }^{1}$ This severe form of abusive head trauma (AHT) is typically associated with retinal hemorrhaging, brain hemorrhaging, and brain swelling, all due to brain acceleration/deceleration during shaking. ${ }^{2}$ Approximately $25 \%$ of SBS injuries are fatal, ${ }^{1}$

and survivors may experience developmental delays, behavioral disorders, seizures, and loss of vision. ${ }^{3-5}$ There is little consensus among physicians and researchers that shaking alone causes the injuries associated with SBS, ${ }^{6}$ and the American Academy of Pediatrics (AAP) and the Center for Disease Control and Prevention (CDC) recommend pediatricians use the term AHT instead of SBS to avoid specifying a mechanism of injury. ${ }^{7,8}$ Despite recommendations from AAP and CDC, medical professionals continue to diagnose SBS, suggesting that both AHT and SBS are relevant diagnoses and definitions of abuse. ${ }^{9}$

Recent trend studies suggest that rates of AHT have decreased, yet there are very few studies on trends of non-fatal SBS over time. ${ }^{10-14}$ SBS is a form of AHT with a unique cause of injury and impetus for abuse among infants and toddlers (shaking and crying, respectively), which suggests that SBS may be more preventable than other forms of abuse. Perpetrators are often caregivers who shake children to stop persistent crying during the first few months after birth. ${ }^{15,16}$ Although medical providers continue to diagnose SBS (Internal Classification of Diseases [ICD] code 995.55), we don't know how diagnosis has shifted over time or whether the ICD code for SBS is capturing all incidences of SBS within hospitals. In 
the absence of this trend data, we cannot know whether prevention efforts have been effective or where future efforts should focus.

In response to this surveillance gap, we examined the National Inpatient Sample (NIS) to compare trends in diagnosed SBS and the diagnosis of SBS-related injuries among children hospitalized for abuse. Hospitalizations are among the most severe cases of non-fatal SBS and provide an opportunity to examine national incidence trends. Similar to the CDC's recommended definitions for measuring probable and definite $\mathrm{AHT}^{7}{ }^{7}$ we measured both possible SBS and confirmed SBS. Confirmed SBS was identified by the presence of the ICD-9 SBS code and we developed a measure for possible SBS to account for hospitalizations that were likely SBS but did not receive an SBS diagnosis. Possible SBS includes two ICD-9 codes related to retinal hemorrhage (362.81), and/or convulsions not associated with a seizure disorder (780.39), which are not included in either CDC definition of AHT.

In all, few, if any, researchers have examined the prevalence of SBS diagnosis codes or how the use of codes are trending over time. In the absence of data on the use of SBS diagnosis codes, we cannot establish the baseline data needed to evaluate SBS prevention programs or diagnostic processes across the nation, leaving children vulnerable to experiencing SBS. Therefore, the purpose of our study was to investigate trends in SBS diagnosis codes among a nationally representative sample of children age three and younger who were hospitalized for abuse. We described the demographic characteristics of children hospitalized for both possible and confirmed SBS, calculated the annual rate of non-SBS abuse and possible and confirmed SBS diagnosis codes, and investigated how trends varied among patient and hospital characteristics.

\section{Method}

\subsection{Sample}

Data from 1998-2014 from the National Inpatient Sample (NIS) ${ }^{17}$ were used for this retrospective study. The NIS is the largest all-payer, inpatient care database in the US and is maintained by the Healthcare Utilization project. We identified our sample of children three years or younger who were hospitalized for abuse using the following ICD-9 diagnosis and external cause of injury codes: Abuse by father/stepfather/boyfriend (E967.0), abuse by mother/step-mother/girlfriend (E967.2), abuse by all other relatives (E967.3 - E967.7), abuse by non-relative (E967.8), neglect (995.52), physical abuse (995.54), psychological abuse (995.51), shaken baby syndrome (995.55), sexual abuse (995.53), and other child abuse not specified $(995.50,995.59$, E967.1, \& E967.9).

Although data from 2015 and 2016 are available, we did not use these data due to the switch from ICD-9CM to ICD-10-CM diagnosis standards in 2015. This change led to substantive shifts in abuse coding, meaning that changes in abuse frequency could not be discerned from changes in the coding standard. All analyses incorporated sampling weights to provide nationally representative estimates. 


\subsection{Measures}

Our analysis included four measures of abusive head trauma within our sample: Non-SBS abuse, confirmed SBS, possible SBS, and total SBS. We defined non-SBS abuse as the absence of diagnosis code 995.55 and confirmed SBS as the presence of diagnosis code 995.55. We constructed possible SBS using codes for physical abuse (995.54) in the presence of a Type 1 internal traumatic brain injury (TBI), retinal hemorrhage (362.81), and/or convulsions not associated with a seizure disorder (780.39). Per the Barell injury matrix, ${ }^{18}$ Type 1 TBI diagnoses include primary intracranial injury, moderate to prolonged loss of consciousness, shaken infant syndrome, or injuries to the optic nerve pathways. We included retinal hemorrhage and convulsions not related to a seizure disorder because they indicate acceleration/deceleration of the head, ${ }^{19}$ yet are not included in the CDC's recommended codes for AHT. ${ }^{7}$ While traumatic brain injury from impact is not precluded, this combination of codes indicates that children were subjected to high-force acceleration injury, characteristic of SBS. Total SBS combines confirmed and possible SBS diagnoses to estimate the actual prevalence of both diagnosed and undiagnosed SBS.

\subsection{Analytic strategy}

We calculated the annual prevalence of SBS hospital diagnoses from 1998 through 2014 for confirmed SBS, possible SBS, and total SBS. Our primary objective was to estimate trends in the probability of confirmed and possible SBS diagnoses. A Chi-square test was used to test for independence of possible and confirmed SBS diagnoses from patient and hospital characteristics. Differences between non-SBS abuse and total SBS were also estimated.

Trends were estimated overall and within categories of age and hospital size. A baseline category logit model was used based on a quasi-likelihood approach (QIC) with an independent working correlation structure. The primary sampling unit in the NIS is the hospital; there are repeated observations within each hospital and across time. To account for the misspecification of the correlation structure, robust standard errors were used (i.e., "sandwich" estimators). Since there is a lack of research on SBS time trends, QIC was used to determine the form of the time trend: linear, quadratic, cubic, or treating time as categorical. A reduction in QIC of two-units was chosen a priori to indicate model preference.

\section{Results}

\subsection{Sample characteristics}

From 1998-2014, there were an estimated 52,562 hospital admissions among children three years of age or younger for Non-SBS abuse and 14,292 for SBS, including 10,083 for confirmed and 4,209 for possible SBS. Possible SBS admissions had a significantly older age distribution than confirmed SBS admissions 
$(p<0.0001 ;$ Table 1$)$. Although not statistically significant, $63.6 \%$ of possible SBS admissions were male compared to $59.7 \%$ of confirmed SBS admissions $(p=0.0749)$. There was no evidence of differences in the racial distributions of possible and confirmed SBS admissions $(p=0.2402)$. Lower income families (1st and 2nd income quartiles) had a higher representation among possible SBS admissions than confirmed SBS admissions (62.1\% vs. $54.2 \% ; p=0.0005)$. Confirmed SBS was most common in large hospitals, while possible SBS was more frequent in medium and small-sized hospital $(p=0.0082)$. Possible SBS was also more frequent than confirmed SBS in urban teaching hospitals when compared to urban non-teaching and rural hospitals $(p=0.0054)$.

\subsection{Time trends in rate of SBS}

In Table 1, we summarize the rates (per 100,000 census population) for non-SBS abuse, possible SBS, confirmed SBS, and total SBS. The overall rate of total SBS diagnoses was $4.5( \pm 0.3)$ for every 100,000 children 3 years old and younger from 1998-2014, whereas the rate of non-SBS abuse was $19.6( \pm 1.0)$. The overall rate of confirmed SBS and possible SBS admissions was $3.8( \pm 0.3)$ and $1.6( \pm 0.1)$, respectively. The annual rate of non-SBS abuse admissions remained fairly stable, with the lowest rate of admissions in 2000 (16.6 \pm 2.0$)$ and the highest rate of admissions in 2010 (25.8 \pm 4.2$)$. However, the rate of admissions for confirmed SBS abuse increased from $3.6( \pm 0.6)$ in 1998 to $5.1( \pm 0.9)$ in 2005, at which point the rate decreased to $1.3( \pm 0.2)$ in 2014 . Conversely, the rate of admissions for possible SBS increased gradually per year. In 1998, the rate of possible SBS admissions was $0.6( \pm 0.2)$ and steadily increased to $2.4( \pm 0.4)$ in 2014 .

Time trends in probability of type of abuse diagnosis. Shown in Figs. 1-3 are the results from the quasilikelihood analyses (solid line and 95\% confidence band) of the time trends in the type of abuse. A significant difference $(p<0.0001)$ was found in the overall trends of the probability of abuse being diagnosed as possible SBS versus confirmed SBS (Fig. 1). Possible SBS gradually increased over time, whereas confirmed SBS slightly increased until 2001 and then decreased from 2002 to 2014 with the trends crossing over in 2011. In 1998, the estimated probability of possible SBS was $2.8 \%$ (95\% Cl: 1.7, 4.6), whereas the chance of confirmed SBS was $18.5 \%$ (95\% Cl: 12.5, 26.4). In 2014, the estimates for possible SBS and confirmed SBS were $9.4 \%(95 \% \mathrm{Cl}: 7.7,11.3)$ and $5.3 \%(95 \% \mathrm{Cl}: 3.4,8.2)$, respectively.

Shown in Fig. 2 are the statistically significant trends for possible SBS versus confirmed SBS by age group: < 1 year old, 1 year old, and 2 or 3 years old. The decrease in the probability of confirmed SBS was largest for infants (< 1 year old), declining from $29.9 \%$ (95\% Cl: $23.2,37.4$ ) in 1998 to $3.2 \%$ (95\% Cl: $1.4 \%$, $7.3 \%$ ) in 2014 , a decrease of $26.7 \%$. Confirmed SBS diagnosis trends for infants ( $<1$ year old) overlapped with possible SBS in 2011. The decrease in confirmed SBS diagnosis trends was more attenuated for young toddlers ( 1 year old) than for older toddlers ( 2 or 3 years old) between 1998 and 2014, decreasing by $8.3 \%$ for young toddlers (15.5\% [95\%: $9.4 \%, 24.4 \%]$ v. $7.2 \%$ [95\% Cl: $3.7 \%, 13.6 \%]$, respectively), and decreasing by $4.0 \%$ for older toddlers (11.2\% [95\%: $5.9 \%, 20.4 \%]$ v. $7.2 \%$ [95\% Cl: $3.2 \%, 13.9 \%$, respectively). The probability of possible SBS increased for all age groups in the same time period. 
Shown in Fig. 3 are the statistically significant trends for possible SBS versus confirmed SBS by hospital size (based on bed count). The decrease in the probability of confirmed SBS was largest for large hospitals between 1998 and 2014 (26.9\% [95\% Cl: 19.4, 35.9] v. 8.7\% [95\% Cl: 5.7\%, 13.0\%]), overlapping with the probability of possible SBS in 2010 . This decrease in confirmed SBS was less attenuated among medium-sized hospitals (17.8\% [95\%: 9.6\%, 30.6\%] v. 8.7\% [95\% Cl: $4.6 \%, 15.4 \%]$ ) than small hospitals (23.7\% [95\%: 13.6\%, 37.3\%] v. 6.8\% (95\% Cl: 3.0\%, 14.4\%]) between 1998 and 2014. In contrast, the probability of possible SBS increased for each hospital size during the same time period.

The trends for confirmed and possible SBS among females and males were similar to the overall trend (Fig. 4). For males, the probability of confirmed SBS increased from 20.5\% (95\% Cl: 13.4\%, 30.1\%) in 1998 to $21.8 \%$ (95\% Cl: $16.8 \%, 27.7 \%)$ in 2000 , and then decreased to $5.0 \%$ (95\% Cl: $2.9 \%, 8.5 \%)$ in 2014 . In contrast, the probability of a male receiving a possible SBS diagnosis increased gradually between 1998 to 2014 (3.1\% [95\% Cl: 1.9, 4.8] v. 11.3\% [95\% Cl: 9.3, 13.5], respectively). For females, the probability of a confirmed SBS diagnosis increased from 15.7\% (95\% Cl: 9.2\%, 25.4\%) in 1998 to $19.2 \%$ (95\% Cl: 14.7\%, $24.6 \%)$ in 2002 , and then decreased to $5.6 \%$ (95\% Cl: $2.9 \%, 10.2 \%)$ in 2014 , whereas the probability of a female receiving a possible SBS diagnosis increased gradually by $4.3 \%$ from 1998 to 2014 (2.4\% [95\% Cl: $1.1 \%, 5.2 \%]$ v. $6.7 \%$ [95\% Cl: $4.8 \%, 9.3 \%]$, respectively).

\section{Discussion}

We investigated seventeen-year trends of possible and confirmed SBS diagnoses among young children admitted to the hospital for abuse. In our study, SBS represented a small subset of overall abuse, which aligns with literature on SBS. ${ }^{10,20,21}$ Consistent with children hospitalized for abuse, our findings indicate that total SBS was more frequent among infants (<1-year-old), boys, or low-income children compared to non-SBS abuse. ${ }^{10,23}$ It is unsurprising that SBS was more frequent among infants than non-SBS abuse, given infants' vulnerability to shaking. ${ }^{3-5,17}$

Our results indicating that girls more frequently received confirmed SBS diagnoses than possible SBS diagnoses approached significance. In contrast, boys more frequently received possible SBS than confirmed SBS diagnoses. Our findings provide context for studies on the relationship between gender and physical abuse diagnoses. When toddlers present with repeat abusive fractures, Ravichandiran and colleagues $^{24}$ reported that physicians initially missed the abuse of boys more often than girls. These findings suggest that physicians may perceive injuries differently among boys and girls, possibly because boys are socialized for rough-and-tumble play and are more prone to accidental injury. ${ }^{25}$

For every subgroup in our study, confirmed SBS diagnoses have declined since 2002, while possible SBS diagnoses have increased. In 2011, possible SBS diagnoses surpassed confirmed SBS and remained higher, i.e., 'the crossover effect.' SBS diagnoses varied by hospital size and type in our study, suggesting a discrepancy in diagnostic processes. Possible SBS diagnoses were more common among urban teaching hospitals and small to medium hospitals than for other hospital types, with the crossover effect occurring as early as 2010 . 
Public opinion and controversy among researchers about SBS may have contributed to demographic variations and the crossover effect of SBS. First, public opinion about SBS appears to have shifted. Both the $C D C$ and $A A P$, which issue best practices for pediatric care, have promoted the use of broad terms like AHT instead of SBS since the mid-2000s. ${ }^{9}$ Mainstream news coverage also has impacted public opinion by focusing on legal cases related to SBS, rather than medical or scientific evidence, and framing SBS as a questionable diagnosis. ${ }^{26}$ Together, media emphasis on legal issues and the AAP's stance on SBS may make physicians more reluctant to diagnose SBS. ${ }^{27}$ Second, the quality and value of SBS research has recently been under scrutiny by some researchers and legal experts, citing inadequate scientific evidence that the injuries typically associated with SBS are caused solely by shaking. ${ }^{28-30}$ Many researchers, however, refute the claim that SBS is diagnosed purely by the presence of retinal hemorrhaging, brain hemorrhaging, and brain swelling, a combination sometimes referred to as the "triad". ${ }^{31-33}$ Narang and Greeley, ${ }^{34}$ for example, emphasized that diagnosing SBS is a complex, context-driven process without reputable diagnosis guidelines.

In all, we contribute to the literature by examining seventeen-year trends of SBS among young children hospitalized for abuse, yet there are limitations. First, our possible SBS measure may not account for all SBS victims who are hospitalized. Physicians use a variety of tools, including available diagnosis codes and a child's history, to diagnose SBS. We chose a combination of codes highly indicative of acceleration/deceleration injury to avoid false-positive diagnoses, but these codes are not exhaustive. Second, our analysis includes no correction for confounders in the estimation of time trends. The lack of research on these trends, however, warranted our approach of estimating simple time trends by subgroup. Finally, we may underestimate actual rates of SBS by excluding SBS victims who were not hospitalized. This surveillance issue is a common problem for all studies on child maltreatment.

\subsection{Implications}

Our findings highlight the difficulty of defining and diagnosing SBS. Many physicians find SBS diagnoses useful, yet the lack of standardized definitions and practice guidelines contribute to the complexity of diagnostic processes. Findley and colleagues ${ }^{25}$ recommend the development of a national registry on SBS and protocols for diagnosing SBS along with alternative explanations for SBS-like injuries. Likewise, we propose that researchers and pediatric medical providers agree to a standardized definition and diagnostic guidelines for SBS, much like the AHT guidelines proposed by CDC, which may help reduce discrepancies in diagnosis and treatment and improve options for surveillance. ${ }^{35,36}$

The distinct biomechanics and etiology of SBS have implications for pediatric education, research, and prevention. Biomechanics research is a main source of evidence about the mechanisms by which shaking injures the pediatric brain. However, biomechanics is not a regular feature of pediatric education. Inclusion of a module on the biomechanics of brain injury could help physicians better understand the underlying mechanical pathways for injury and related symptoms and outcomes for infant-shaking injuries, as well as injuries like sports concussions. Investment in biomechanical research, which has not 
been prominent in injury prevention research for two decades, would also advance knowledge of causal mechanisms.

Finally, our finding that total SBS has increased over time suggests that prevention efforts have not been far-reaching enough to address the scope of the problem. Most prevention programs focus on raising awareness about normal infant crying and the dangers of shaking a baby, as well as improving caregivers' response to crying. ${ }^{37}$ Results from these programs are promising, suggesting that prevention programs can improve parental knowledge about crying behaviors and may help reduce emergency department visits for prolonged crying. ${ }^{38}$ The Period of PURPLE Crying, for example, is a prevention campaign designed by the National Center on Shaken Baby Syndrome and well known for its empirical support. ${ }^{34,35}$ Given our findings and the evidence on prevention programs, prevention programs should be expanded to more locations and offered to more expecting parents.

\section{Conclusion}

SBS is a severe form of child physical abuse with lifelong consequences for survivors. Our study findings demonstrated that confirmed SBS has decreased since 2002 while possible SBS has increased. The discrepancy between trends in possible and confirmed SBS suggests difference in norms for diagnosing SBS. Future research should investigate diagnostic processes for SBS and the reasons why cases are diagnosed as SBS or not. Stopping the cycle of SBS requires that society adopt policies and programs to support new parents who are overwhelmed and susceptible to shaking their infants. In the absence of systemic changes, our youngest and most vulnerable members of society will continue experiencing SBS, a highly preventable public health problem.

\section{Abbreviations}

AAP: American Academy of Pediatrics; AHT: Abusive head trauma; CDC: Center for Disease Control and Prevention; Cl: Confidence interval; ICD9-CM: International classification of diseases, clinical modification, ninth revision; NIS: National Inpatient Sample; SBS: Shaken baby syndrome; TBI: Traumatic brain injury; QIC: Quasi-likelihood information criterion.

\section{Declarations}

\section{Availability of data and materials}

The data that support the findings of this study are available for purchase from the Healthcare Utilization Project. Data are available, however, from the authors upon reasonable request and with permission of the Healthcare Utilization Project.

\section{Authors' contributions}


$A C, R O, B B$, and CPA contributed significantly to the study conceptualization and design. AC contributed to manuscript writing. CPA and BB acquired the data, performed data cleaning and analysis, and contributed to manuscript writing. RO provided editing and guidance throughout manuscript revisions. MR conducted the literature review and contributed to manuscript writing. All authors read and approved the final manuscript.

\section{Ethics approval and consent to participate}

Not applicable. NIS is a publicly available dataset.

\section{Consent for publication}

Not applicable.

\section{Competing interests}

The authors declare that they have no competing interests.

\section{Funding}

Not applicable.

\section{Acknowledgements}

Not applicable.

\section{References}

1. National Center on Shaken Baby Syndrome. Learn More. https://dontshake.org/learn-more. Published 2019. Accessed May 11, 2019.

2. Bandak FA. Shaken baby syndrome: A biomechanics analysis of injury mechanisms. Forensic Science International. 2005;151(1):71-79. doi:10.1016/j.forsciint.2005.02.033

3. King WJ, MacKay M, Sirnick A. Shaken baby syndrome in Canada: Clinical characteristics and outcomes of hospital cases. CMAJ. 2003;168(2):155-159. http://www.cmaj.ca/content/cmaj/168/2/155.full.pdf.

4. Laurent-Vannier A, Toure $H$, Vieux E, Brugel D, Chevignard M. Long-term outcome of the shaken baby syndrome and medicolegal consequences: A case report. Annals of Physical and Rehabilitation Medicine. 2009;52(5):436-447. doi:10.1016/j.rehab.2009.03.001.

5. Lind K, Toure H, Brugel D, Meyer P, Laurent-Vannier A, Chevignard M. Extended follow-up of neurological, cognitive, behavioral and academic outcomes after severe abusive head trauma. Child Abuse \& Neglect. 2016;51:358-367. doi:10.1016/j.chiabu.2015.08.001.

6. Schiks L, Dankelman J, Loeve A. Thresholds for the assessment of inflicted head injury by shaking trauma in infants: A systematic review. Forensic Science International. 2020;306. 
https://doi.org/10.1016/j.forsciint.2019.110060.

7. Center for Disease Control and Prevention (2012). Pediatric abusive head trauma: Recommended definitions for public health surveillance and research. Retrieved from https://www.cdc.gov/violenceprevention/pdf/pedheadtrauma-a.pdf.

8. Christian CW, Block R. Abusive head trauma in infants and children. Pediatrics. 2009;123(5):14091411. doi:10.1542/peds.2009-0408.

9. Narang SK, Estrada C, Greenberg S, Lindberg D. Acceptance of shaken baby syndrome and abusive head trauma as medical diagnoses. The Journal of Pediatrics. 2016;177:273-278. doi:10.1016/j.jpeds.2016.06.036.

10. Wood JN, French B, Fromkin J, et al. Association of pediatric abusive head trauma rates with macroeconomic indicators. Academic Pediatrics. 2016;16(3):224-232. doi:10.1016/j.acap.2015.05.008.

11. Shanahan ME, Zolotor AJ, Parrish JW, Barr RG, Runyan DK. National, regional, and state abusive head trauma: Application of the CDC Algorithm. Pediatrics. 2013;132(6). doi:10.1542/peds.20132049

12. Niederkrotenthaler T, Xu L, Parks SE, Sugerman DE. Descriptive factors of abusive head trauma in young children-United States, 2000-2009. Child Abuse \& Neglect. 2013;37(7):446-455. doi:10.1016/j.chiabu.2013.02.002

13. Yu YR, Demello AS, Greeley CS, Cox CS, Naik-Mathuria BJ, Wesson DE. Injury patterns of child abuse: Experience of two Level 1 pediatric trauma centers. Journal of Pediatric Surgery. 2018;53(5):10281032. doi:10.1016/j.jpedsurg.2018.02.043

14. Spies E, Klevens J. Fatal abusive head trauma among children aged $<5$ years- United States, 1999 2014. MMWR Morb Mortal Wkly Rep. 2016;65(20):505-509.

15. Lee C, Barr RG, Catherine N, Wicks A. Age-related incidence of publicly reported shaken baby syndrome cases: Is crying a trigger for shaking? Journal of Developmental \& Behavioral Pediatrics. 2007;28(4):288-293. doi:10.1097/dbp.0b013e3180327b55.

16. Barr RG. Preventing abusive head trauma resulting from a failure of normal interaction between infants and their caregivers. Proceedings of the National Academy of Sciences. 2012;109:1729417301. doi:10.1073/pnas.1121267109

17. Agency for Healthcare Research and Quality (AHRC). Healthcare cost and utilization project national inpatient sample. 2012. Rockville, Maryland.

18. Bergen G, Chen LH, Warner M, Fingerhut LA. Injury in the United States: 2007 Chartbook. Hyattsville, MD: National Center for Health Statistics. 2008. https://www.cdc.gov/nchs/data/misc/injury2007.pdf. Accessed May 21, 2019.

19. Hymel KP, Bandak F, Partington M, Winston K. Abusive head trauma? A biomechanics-based approach. Child Maltreatment. 1998;3(2):116-128.

20. Barr RG, Trent RB, Cross J. Age-related incidence curve of hospitalized Shaken Baby Syndrome cases: Convergent evidence for crying as a trigger to shaking. Child Abuse \& Neglect. 2006;30(1):7- 
16. doi:10.1016/j.chiabu.2005.06.009.

21. Leventhal JM, Martin KD, Gaither JR. Using US Data to Estimate the Incidence of Serious Physical Abuse in Children. Pediatrics. 2012;129(3):458-464. doi:10.1542/peds.2011-1277d.

22. U.S. Department of Health \& Human Services, Administration for Children and Families. Child Maltreatment 2017. https://www.acf.hhs.gov/cb/research-data-technology/ statistics-research/childmaltreatment. Published 2019. Accessed July 21, 2019.

23. Russo CA, Hambrick MM, Owens PL. Hospital stays related to child maltreatment, 2005. HCUP Statistical Brief \#49. April 2008. Agency for Healthcare Research and Quality, Rockville, MD. http://www.hcup-us.ahrq.gov/reports/statbriefs/sb49.pdf. Accessed October 9, 2018.

24. Ravichandiran N, Schuh S, Bejuk M, et al. Delayed identification of pediatric abuse-related fractures. Pediatrics. 2009;125(1):60-66. doi:10.1542/peds.2008-3794.

25. Hagan LK, Kuebli J. Mothers and fathers socialization of preschoolers physical risk taking. Journal of Applied Developmental Psychology. 2007;28(1):2-14. doi:10.1016/j.appdev.2006.10.007

26. Hennink-Kaminski HJ, Dougall EK. Myths, mysteries, and monsters: When shaken babies make the sews. Social Marketing Quarterly. 2009;15(4):25-48. doi:10.1080/15245000903348764.

27. Cowley LE, Maguire S, Farewell DM, Quinn-Scoggins HD, Flynn MO, Kemp AM. Factors influencing child protection professionals' decision-making and multidisciplinary collaboration in suspected abusive head trauma cases: A qualitative study. Child Abuse \& Neglect. 2018;82:178-191. doi:10.1016/j.chiabu.2018.06.009.

28. Findley KA, Barnes PD, Moran DA, Squier W. Shaken baby syndrome, abusive head trauma, and actual innocence: Getting it right. Hous J Health L \& Pol'y. 2011;12(2):209-312.

29. Lynøe N, Elinder G, Hallberg B, Rosen M, Sundgren P, Eriksson A. Insufficient evidence for 'shaken baby syndrome'- A systematic review. Acta Paediatrica. 2017;106:1021-1027. doi:DOI:10.1111/apa.13760.

30. Lyons G. Shaken baby syndrome: A questionable scientific syndrome and a dangerous legal concept. Utah L Rev. 2003;1109(3):1109-1132.

31. Bilo RA, Banaschak S, Herrmann B, et al. Using the table in the Swedish review on shaken baby syndrome will not help courts deliver justice. Acta Paediatrica. 2017;106(7):1043-1045. doi:10.1111/apa.13857.

32. Laurent-Vannier A, Adamsbaum C, Raul J-S, Rey-Salmon C, Rambaud C. Flawed Swedish study on traumatic shaking is already being used by defence lawyers and its findings must be ignored. Acta Paediatrica. 2018;107(12):2048-2050. doi:10.1111/apa.14564.

33. Strouse PJ. Shaken baby syndrome is real. Pediatric Radiology. 2018;48(8):1043-1047. doi:10.1007/s00247-018-4158-0.

34. Narang SK, Greeley CS. Lynøe et al. - \#theRestoftheStory. Acta Paediatrica. 2017;106(7):1047-1049. doi:10.1111/apa.13858. 
35. Paine CW, Scribano PV, Localio R, Wood JN. Development of guidelines for skeletal survey in young children with intracranial hemorrhage. Pediatrics. 2016;137(4). doi:10.1542/peds.2015-3024.

36. Kim PT, Mccagg J, Dundon A, Ziesler Z, Moody S, Falcone RA. Consistent screening of admitted infants with head injuries reveals high rate of nonaccidental trauma. Journal of Pediatric Surgery. 2017;52(11):1827-1830. doi:10.1016/j.jpedsurg.2017.02.014.

37. Lopes NRL, Williams LCDA. Pediatric abusive head trauma prevention initiatives: A literature review. Trauma, Violence, \& Abuse. 2016;19(5):555-566. doi:10.1177/1524838016675479.

38. Barr RG, Rajabali F, Aragon M, Colbourne M, Brant R. Education about crying in normal infants is associated with a reduction in pediatric emergency room visits for crying complaints. Journal of Developmental \& Behavioral Pediatrics. 2015;36(4):252-257. doi:10.1097/dbp.0000000000000156.

\section{Tables}


Table 1. National hospital admissions for children up to three years, 1998 - 2014, patient- and hospital-level descriptive statistics, N (\%), by type of abuse.

\begin{tabular}{|c|c|c|c|c|c|c|}
\hline Variable & $\begin{array}{c}\text { Possible SBS } \\
4,209(29.4) \\
\end{array}$ & $\begin{array}{c}\text { Confirmed SBS } \\
10,083(70.6) \\
\end{array}$ & $\begin{array}{c}\text { Chi-square } \\
\text { P value }\end{array}$ & $\begin{array}{c}\text { Total SBS } \\
14,292(21.4) \\
\end{array}$ & $\begin{array}{c}\text { Non-SBS Abuse } \\
52,562(79.2) \\
\end{array}$ & $\begin{array}{c}\text { Chi-square } \\
\text { P value }\end{array}$ \\
\hline \multicolumn{7}{|l|}{ Rates (per 100,00 population) } \\
\hline Total rate of abuse & $1.6( \pm 0.1)$ & $3.8( \pm 0.3)$ & --- & $5.4( \pm 0.3)$ & $19.6( \pm 1.0)$ & --- \\
\hline \multicolumn{7}{|l|}{ Rate of abuse per year } \\
\hline 1998 & $0.6( \pm 0.2)$ & $3.6( \pm 0.6)$ & --- & $4.1( \pm 0.7)$ & $17.8( \pm 2.5)$ & --- \\
\hline 1999 & $1.1( \pm 0.3)$ & $6.4( \pm 1.0)$ & --- & $7.5( \pm 1.2)$ & $20.1( \pm 2.3)$ & -- \\
\hline 2000 & $0.6( \pm 0.2)$ & $4.6( \pm 0.8)$ & -- & $5.2( \pm 0.9)$ & $16.6( \pm 2.0)$ & -- \\
\hline 2001 & $1.0( \pm 0.3)$ & $4.5( \pm 0.8)$ & --- & $5.5( \pm 1.1)$ & $19.1( \pm 3.1)$ & --- \\
\hline 2002 & $0.7( \pm 0.2)$ & $4.3( \pm 0.8)$ & --- & $5.0( \pm 0.9)$ & $17.5( \pm 2.3)$ & --- \\
\hline 2003 & $1.1( \pm 0.3)$ & $5.0( \pm 0.8)$ & --- & $6.1( \pm 1.0)$ & $18.2( \pm 2.6)$ & -- \\
\hline 2004 & $1.3( \pm 0.4)$ & $4.8( \pm 0.9)$ & -- & $6.1( \pm 1.2)$ & $18.1( \pm 2.7)$ & -- \\
\hline 2005 & $2.0( \pm 0.6)$ & $5.1( \pm 0.9)$ & --- & $7.1( \pm 1.3)$ & $22.7( \pm 3.6)$ & -- \\
\hline 2006 & $1.3( \pm 0.4)$ & $4.5( \pm 0.8)$ & --- & $5.9( \pm 1.0)$ & $17.9( \pm 2.5)$ & -- \\
\hline 2007 & $2.0( \pm 0.6)$ & $3.8( \pm 0.7)$ & --- & $5.8( \pm 1.1)$ & $18.7( \pm 3.5)$ & -- \\
\hline 2008 & $1.2( \pm 0.4)$ & $3.8( \pm 0.6)$ & --- & $5.0( \pm 0.9)$ & $17.3( \pm 2.9)$ & --- \\
\hline 2009 & $1.9( \pm 0.5)$ & $3.1( \pm 0.6)$ & --- & $5.0( \pm 1.0)$ & $17.3( \pm 2.8)$ & -- \\
\hline 2010 & $1.9( \pm 0.5)$ & $3.6( \pm 0.6)$ & -- & $5.6( \pm 0.9)$ & $25.8( \pm 4.2)$ & -- \\
\hline 2011 & $2.6( \pm 0.7)$ & $1.5( \pm 0.3)$ & --- & $4.1( \pm 0.9)$ & $18.8( \pm 3.8)$ & -- \\
\hline 2012 & $2.3( \pm 0.4)$ & $2.2( \pm 0.3)$ & --- & $4.4( \pm 0.5)$ & $21.4( \pm 2.0)$ & --- \\
\hline 2013 & $2.5( \pm 0.4)$ & $2.0( \pm 0.3)$ & --- & $4.5( \pm 0.5)$ & $23.0( \pm 2.0)$ & --- \\
\hline 2014 & $2.4( \pm 0.4)$ & $1.3( \pm 0.2)$ & -- & $3.7( \pm 0.4)$ & $22.5( \pm 2.0)$ & -- \\
\hline \multicolumn{7}{|l|}{ Patient Characteristics } \\
\hline \multicolumn{7}{|l|}{ Age } \\
\hline$<1$ & $2,744(65.2)$ & $8,162(80.9)$ & $<0.0001$ & $10,906(76.3)$ & $33,428(63.6)$ & $<0.0001$ \\
\hline 1 & $700(16.6)$ & 1,160 (11.5) & & $1,860(13)$ & 8,705 (16.6) & \\
\hline $2 / 3$ & $765(18.2)$ & $761(7.5)$ & & $1,526(10.7)$ & $10,429(19.8)$ & \\
\hline \multicolumn{7}{|l|}{ Sex } \\
\hline Female & $1,530(36.4)$ & $4,067(40.3)$ & 0.0749 & $5,597(39.2)$ & $22,486(42.8)$ & 0.0007 \\
\hline Male & $2,679(63.6)$ & $6,016(59.7)$ & & $8,695(60.8)$ & $30,075(57.2)$ & \\
\hline \multicolumn{7}{|l|}{ Race } \\
\hline Asian or Pacific Islander & $63(1.5)$ & $260(2.6)$ & 0.2402 & $323(2.3)$ & $467(0.9)$ & $<0.0001$ \\
\hline Black & $853(20.3)$ & 1,992 (19.8) & & 2,845 (19.9) & $12,774(24.3)$ & \\
\hline Hispanic & $718(17.1)$ & $1,614(16)$ & & 2,332 (16.3) & $9,261(17.6)$ & \\
\hline Native American & $58(1.4)$ & $84(0.8)$ & & $142(1)$ & $500(1)$ & \\
\hline Other & $310(7.4)$ & $574(5.7)$ & & $884(6.2)$ & $2,632(5)$ & \\
\hline White & $2,206(52.4)$ & $5,559(55.1)$ & & $7,765(54.3)$ & $26,928(51.2)$ & \\
\hline \multicolumn{7}{|l|}{ Income } \\
\hline 1st Quartile & $1,262(30)$ & $2,558(25.4)$ & 0.0005 & $3,820(26.7)$ & $18,216(34.7)$ & $<0.0001$ \\
\hline 2nd Quartile & 1,349 (32.1) & 2,906 (28.8) & & $4,255(29.8)$ & $15,490(29.5)$ & \\
\hline 3rd Quartile & $1,037(24.6)$ & $2,572(25.5)$ & & $3,609(25.3)$ & $11,703(22.3)$ & \\
\hline 4th Quartile & 560 (13.3) & $2,047(20.3)$ & & $2,607(18.2)$ & 7,153 (13.6) & \\
\hline \multicolumn{7}{|l|}{ Hospital Characteristics } \\
\hline \multicolumn{7}{|l|}{ Hospital Type (since 2008) } \\
\hline Government, nonfederal & $331(14.4)$ & $413(14.9)$ & 0.0677 & $744(15.9)$ & $3,641(14.6)$ & 0.6438 \\
\hline Private, investor-owned & $98(4.3)$ & $243(8.7)$ & & $341(6.7)$ & $1,541(6.7)$ & \\
\hline Private, not-for-profit & $1,874(81.4)$ & $2,123(76.4)$ & & 3,997 (77.4) & $17,700(78.6)$ & \\
\hline \multicolumn{7}{|l|}{ Hospital Size (bed count) } \\
\hline Large & $2,380(57.4)$ & $6,704(66.7)$ & 0.0082 & $9,084(64.0)$ & $32,545(62.5)$ & 0.4798 \\
\hline Medium & $1,111(26.8)$ & 1,944 (19.3) & & $3,055(21.5)$ & $12,063(23.2)$ & \\
\hline Small & 656 (15.8) & 1,398 (13.9) & & $2,054(14.5)$ & $7,455(14.3)$ & \\
\hline \multicolumn{7}{|l|}{ Hospital Location / Teaching } \\
\hline Rural & $45(1.1)$ & $328(3.3)$ & 0.0054 & $373(2.6)$ & $3,294(6.3)$ & $<0.0001$ \\
\hline Urban Non-Teaching & $263(6.2)$ & $1,258(12.5)$ & & $1,521(10.6)$ & $6,808(13)$ & \\
\hline Urban Teaching & 3,901 (92.7) & $8,497(84.3)$ & & $12,398(86.7)$ & $42,460(80.8)$ & \\
\hline
\end{tabular}




\begin{tabular}{|c|c|c|c|c|c|c|}
\hline spital Reg & & & & & & \\
\hline Midwest & $1,210(28.7)$ & $2,707(26.8)$ & 0.1281 & 3,917 (27.4) & 13,928 (26.5) & 0.2049 \\
\hline Northeast & 436 (10.4) & $1,518(15.1)$ & & 1,954 (13.7) & $8,459(16.1)$ & \\
\hline South & 1,675 (39.8) & $4,177(41.4)$ & & $5,852(40.9)$ & $20,144(38.3)$ & \\
\hline West & 888 (21.1) & 1,681 (16.7) & & 2,569 (18) & $10,029(19.1)$ & \\
\hline
\end{tabular}

\section{Figures}

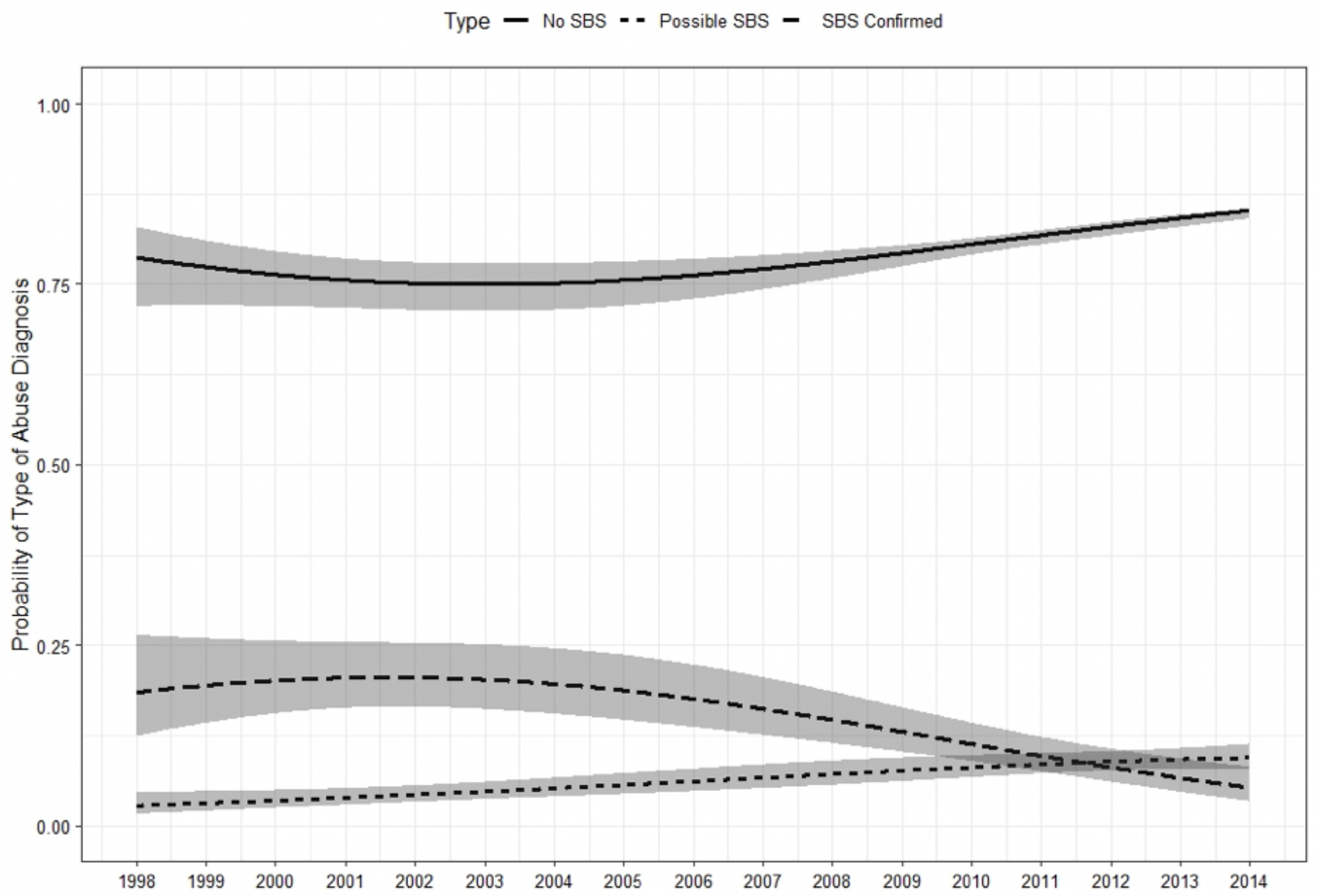

Figure 1

Overall time trend in the probability of diagnosing abuse as Confirmed SBS, Possible SBS, and Non-SBS abuse. 
Type - No SBS - - Possible SBS - SBS Confirmed
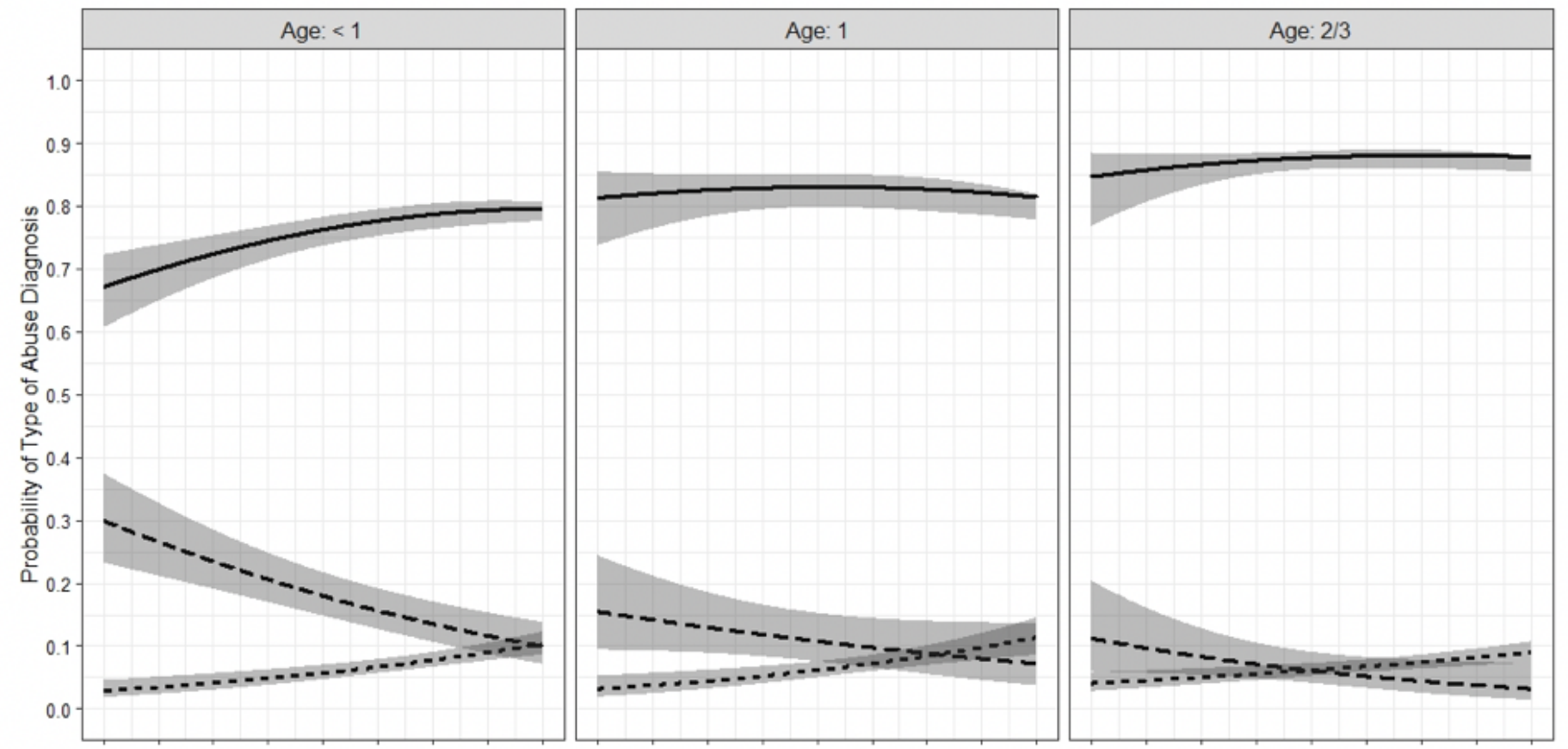

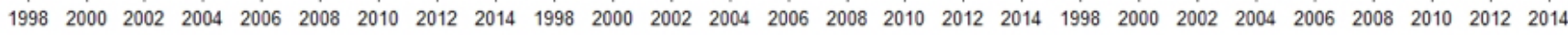

\section{Figure 2}

Overall time trend in the probability of diagnosing abuse as Confirmed SBS, Possible SBS, and Non-SBS abuse by age of patient. 
Type - No SBS - - Possible SBS - ' SBS Confirmed
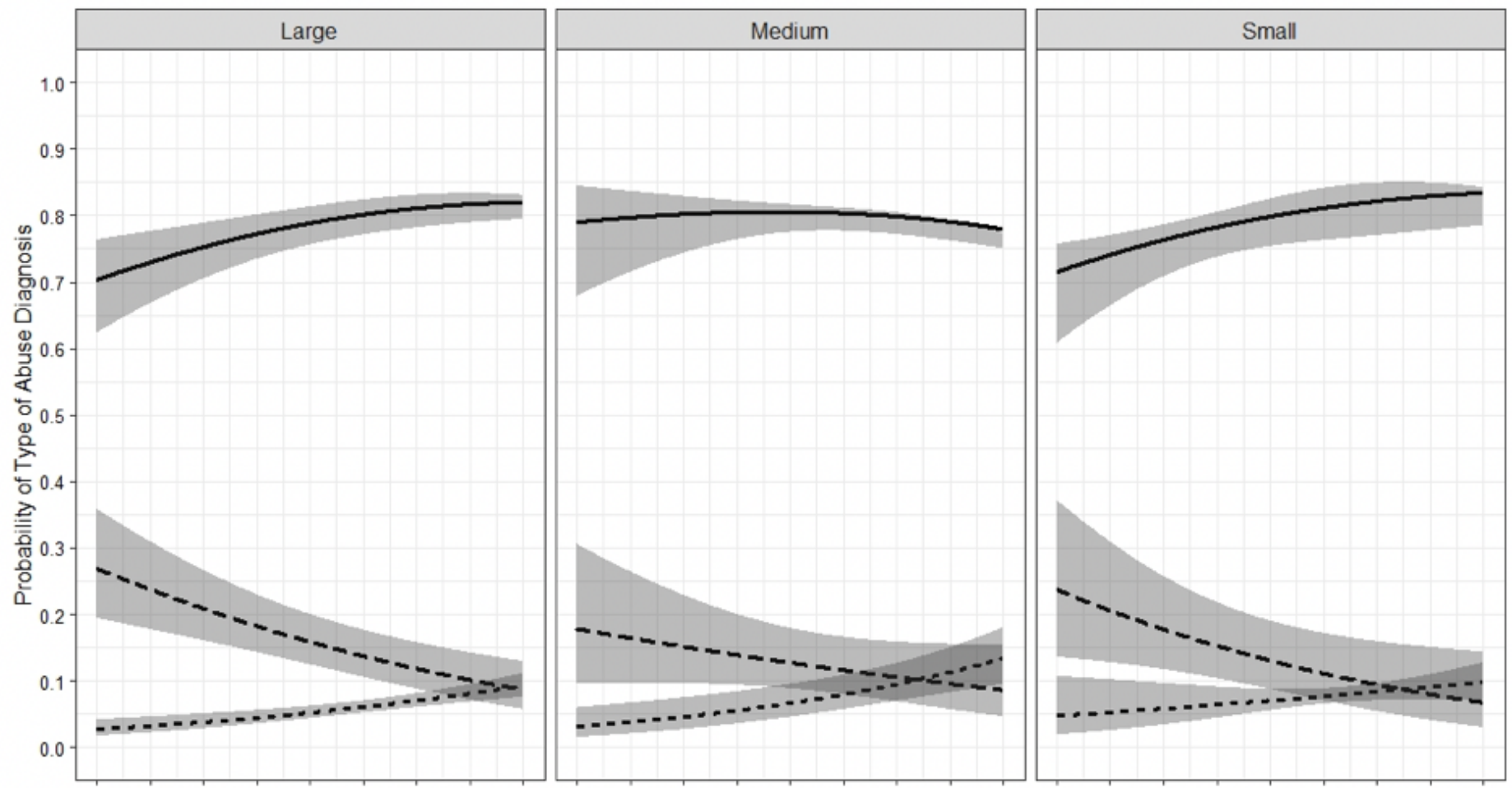

$19982000200220042006200820102012 \quad 2014 \quad 199820002002200420062008201020122014 \quad 199820002002 \quad 2004200620082010 \quad 2012 \quad 2014$

\section{Figure 3}

Overall time trend in the probability of diagnosing abuse as Confirmed SBS, Possible SBS, and Non-SBS abuse by hospital bed-size. 
Type - No SBS - - Possible SBS - ' SBS Confirmed
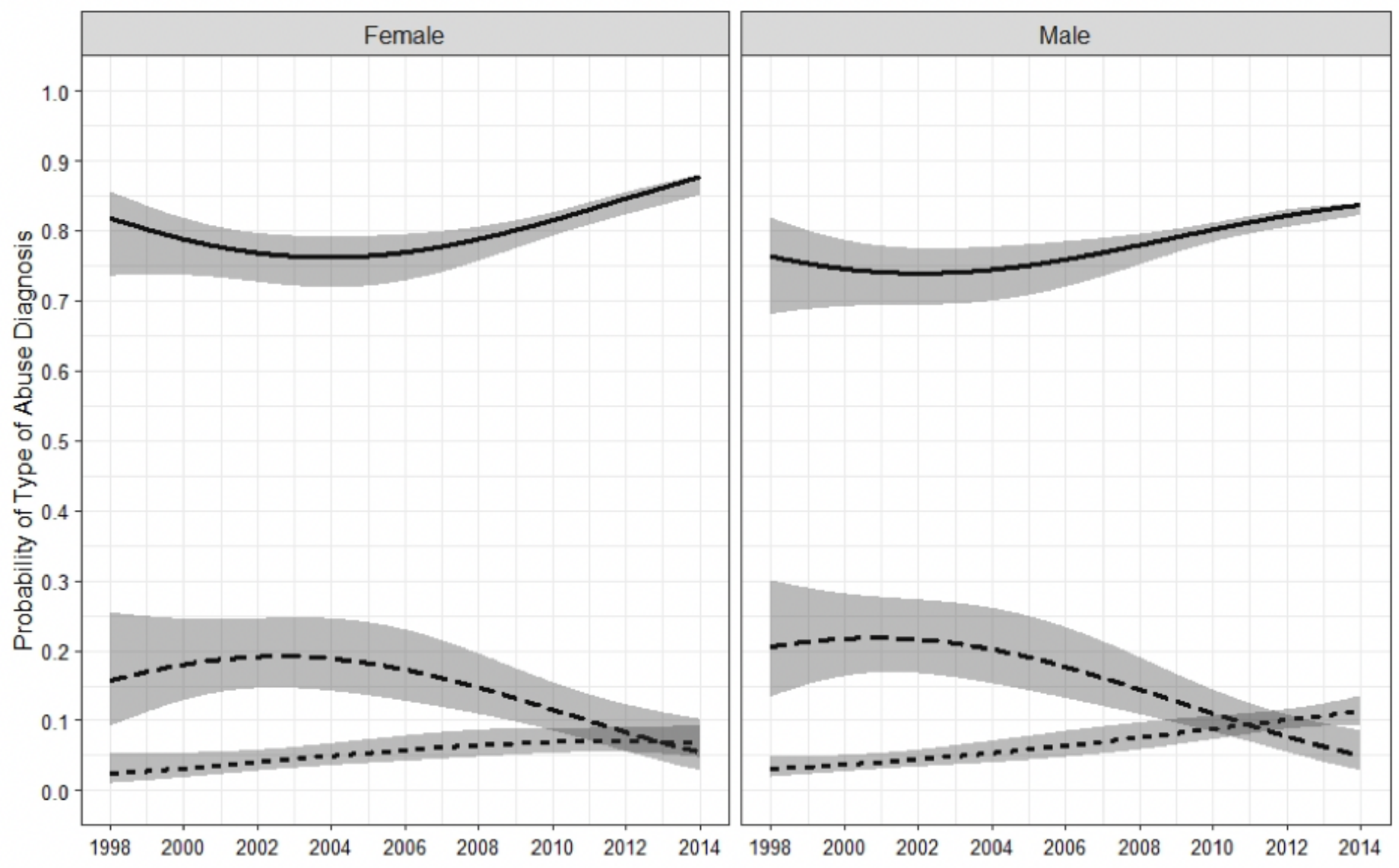

Figure 4

Overall time trend in the probability of diagnosing abuse as Confirmed SBS, Possible SBS, and Non-SBS abuse by sex of patient. 